MRS Advances (C) 2019 Materials Research Society. This is an Open Access article, distributed under the terms of the Creative Commons Attribution licence (http:// creativecommons.org/licenses/by/4.0/), which permits unrestricted re-use, distribution, and reproduction in any medium, provided the original work is properly cited.

DOI: 10.1557/adv.2019.125

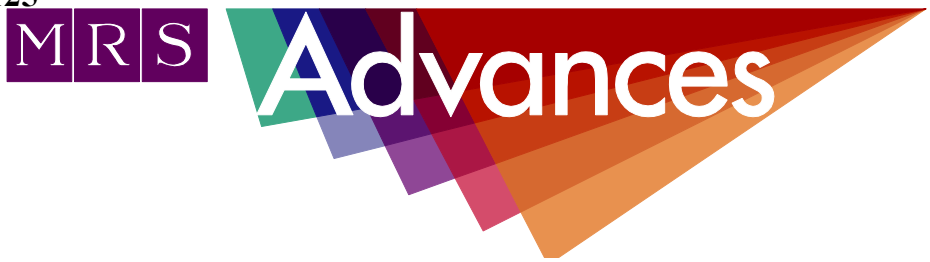

\title{
The Role of the Binder/Solvent Pair on the Electrochemical Performance of Aluminium Batteries
}

\author{
Jasmin Smajic ${ }^{1}$, Amira Alazmi ${ }^{1}$, Pedro M. F. J. Costa ${ }^{1 *}$
}

${ }^{1}$ King Abdullah University of Science and Technology, Physical Science and Engineering Division, Thuwal 23955-6900, Saudi Arabia

ABSTRACT

In energy storage systems, every component that makes up an electrode can greatly affect the electrochemical performance. One example includes the so-called "binders" used in secondary batteries. Herein, we compare the influence of using polyvinylidene fluoride $(P V D F)$ or sodium carboxymethyl cellulose $(C M C)$ on the electrochemical performance of an aluminium chloride battery $(A C B)$ system. The active material of the cathode was a reduced graphene oxide dried under supercritical conditions (RGOCPD). Interestingly, while PVDF enabled one of the highest capacities reported for ACBs, the CMC resulted in a significant degradation of the cell's performance.

\section{INTRODUCTION}

A battery electrode commonly consists of four parts that must act synergistically to enable reliable and optimized performance: current collectors, electrochemically active materials, conductive additives and binders. The latter play a crucial role by binding the active material and the conductive additive particles and promoting adhesion to the current collector. Hence, the structural integrity of the electrode is ensured. The binder is usually dissolved in a solvent, therefore, a proper choice of the binder/solvent pair can greatly influence the electrode's electrochemical response. Generally, polyvinylidene fluoride (PVDF) and n-methyl-2-pyrrolidone (NMP) are accepted as the default binder/solvent pair, respectively, for lithium-ion batteries. [1] However, it seems logical that each and every battery should be optimized with regards to their specific components. For instance, sodium carboxymethyl cellulose (CMC) is a binder that presents some attractive advantages over PVDF such as being soluble in water, biocompatible and having stronger adhesive and dispersing properties. 
In a previous study, [2] we reported that the pore size distribution of the active material had a decisive effect on the electrochemical performance of aluminum chloride batteries (ACBs). Here, we show that the choice of the binder/solvent pair is another factor to carefully assess in the optimization of ACBs.

\section{EXPERIMENTAL DETAILS}

\section{Materials synthesis and characterization}

To prepare the electrolyte, [EMIm]Cl was dehydrated in a tube furnace (in vacuo, at $70{ }^{\circ} \mathrm{C}$ and for 16 hours $)$ and taken to an Ar-filled glove box $\left(<0.5 \mathrm{ppm} \mathrm{O}_{2},<0.5\right.$ ppm $\mathrm{H}_{2} \mathrm{O}$ ). Then, anhydrous $\mathrm{AlCl}_{3}$ (Alfa Aesar) was slowly added (note: exothermic reaction) to the $[\mathrm{EMIm}] \mathrm{Cl}$ in a $1.3: 1$ molar ratio to produce the electrolyte $\mathrm{AlCl}_{3}:[\mathrm{EMIm}] \mathrm{Cl}$. The clear light yellow liquid was stirred at $1000 \mathrm{RPM}$, for 30 minutes and left to stand. To prepare the active material, reduced graphene oxide (RGO), graphite powder (99\%, crystalline, 325 mesh, Alfa Aesar) was oxidized and exfoliated using the improved Hummers' method. [3] The brownish product was subsequently dried in supercritical $\mathrm{CO}_{2}$, for 12 hours, followed by hydrothermal treatment at $180{ }^{\circ} \mathrm{C}$, for 24 hours. Similar to the parent GO, the resulting RGO was dried in a critical point dryer (CPD) and dubbed RGOCPD.

The structural analysis of the precursors and electrode materials was performed by powder X-ray diffraction (XRD) on a Bruker D8 ADVANCE $(\mathrm{Cu} \mathrm{K \alpha}$ radiation, $\lambda=$ $1.54 \AA, 5 \mathrm{deg} \min ^{-1}$ and $0.02^{\circ}$ increments). Raman spectra were recorded on a Witec Alpha 300RA with a $532 \mathrm{~nm}$ solid-state laser. The Fourier transform infrared (FTIR) analysis was carried out on a Thermo Fischer Scientific Nicolet iS10 spectrometer, in transmission mode and using $\mathrm{KBr}$ pellets.

\section{$\underline{\text { Electrochemical measurements }}$}

Electrochemical measurements were done on a Bio-Logic VMP3 potentiostat, at room temperature and using Swagelok two-electrode cells assembled inside an Arfilled glove box (LABstar, MBraun). The cathode's active material was RGOCPD, and Al foils $(99.999 \%, 0.25 \mathrm{~mm}$, Sigma-Aldrich) were used as the counter and reference electrodes. The cathode was fabricated by mixing the active material ( $80 \mathrm{wt} \%)$, a binder (PVDF or CMC; $10 \mathrm{wt} \%)$ and acetylene black (10 wt \%) in either NMP or deionized water. The obtained slurry was transferred into a vial, stirred for 24 hours and spread on a $\mathrm{Nb}$ foil (current collector, $0.25 \mathrm{~mm}$ ). Cathodes with PVDF are dubbed PVDF/RGOCPD and those with CMC, CMC/RGOCPD. Borosilicate microfiber filters (grade GF/F, Whatman) were used as separators. These were desiccated in a tube furnace (in vacuo, at $150{ }^{\circ} \mathrm{C}$ and for 16 hours) and introduced into the glove box, where they were soaked with $10 \mu \mathrm{l}$ of the electrolyte. After assembling, the cells were left to stand for 16 hours (in open circuit conditions) to properly wet the electrodes with the electrolyte. Next, they were subjected to cyclic voltammetry (CV) activation step, employing over 500 cycles at $20 \mathrm{mV} \mathrm{s}^{-1}(0.01-2.2 \mathrm{~V})$. The galvanostatic charge and discharge experiments were carried out at $100 \mathrm{~mA} \mathrm{~g}^{-1}(0.01-2.2 \mathrm{~V})$. All capacities are reported per mass of the active material. 


\section{RESULTS \& DISCUSSION}

Whilst the CV of the PVDF/RGOCPD exhibited only faint and broad redox couples - indicating a charge storage mechanism that is mostly surface-based, the one for CMC/RGOCPD showed more distinguishable peaks, implying an increased contribution of Faradaic electrode-electrolyte charge-transfers (Fig. 1a). The profiles in Fig. 1b confirmed this difference since voltage plateaus were visible only for the CMC/RGOCPD. Both cathodes showed good cycling stability, but the CMC-based one had a much lower specific discharge capacity (Fig. 1c). In fact, its initial discharge capacity was merely $23 \mathrm{mAh} \mathrm{g}^{-1}$ (for a Coulombic efficiency of 52\%) while for the PVDF/RGOCPD it was $171 \mathrm{mAh} \mathrm{g}^{-1}$ (Coulombic efficiency of $81 \%$ ). Of note, the performance of the CMC-based cathode improved after 50 cycles (to $46 \mathrm{mAh} \mathrm{g}^{-1}$, for a Coulombic efficiency of $71 \%$ ). This stands in contrast to the PVDF/RGOCPD which, at the same point, had $155 \mathrm{mAh} \mathrm{g}^{-1}$ of discharge capacity and constant Coulombic efficiency. None of the binders contributed to the overall capacity as their intrinsic discharge capacity is negligible, while carbon black rapidly settles to $<5 \mathrm{mAh} \mathrm{g}^{-1}$ (Fig. 1d).

a)

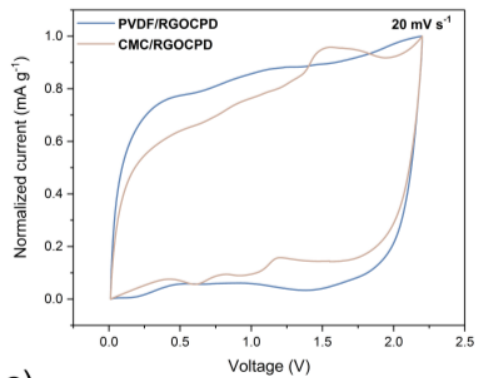

c)

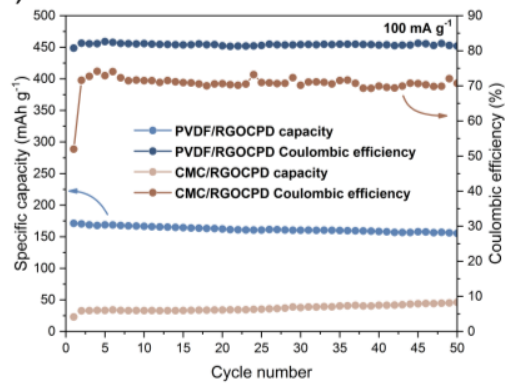

b)

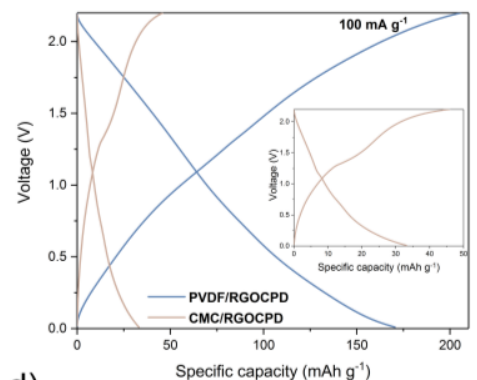

d)

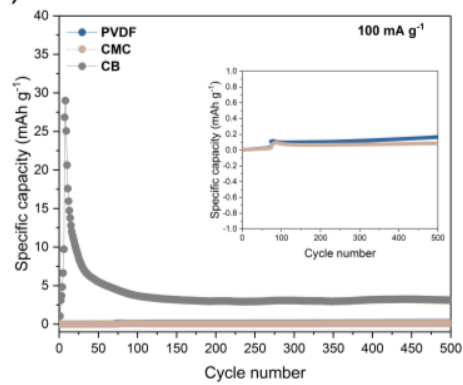

Figure 1. Electrochemical performance of PVDF/RGOCPD and CMC/RGOCPD: a) $\mathrm{CV}$ at $20 \mathrm{mV} \mathrm{s}^{-1}$; b) voltage profiles at $100 \mathrm{~mA} \mathrm{~g}^{-1}$; c) cyclic stability at $100 \mathrm{~mA} \mathrm{~g}^{-1}$; d) binders and carbon black capacity contribution.

The RGOCPD is a mesoporous material that could experience structural collapse when subjected to surface tension induced by solvents. If so, this would bring its electrochemical response closer to that of its graphite precursor, by lowering its discharge capacity and showing stronger intercalation peaks in the cyclic voltammograms (as seen in Fig. 1a for CMC/RGOCPD). [1] To verify this hypothesis, structural and textural changes in the precursor (graphite and RGOCPD) and electrode (PVDF/RGOCPD and CMC/RGOCPD) materials, were studied with Raman and powder XRD. In addition, binder-free samples of RGOCPD exposed to NMP or water were prepared and characterised (dubbed NMP/RGOCPD and Water/RGOCPD). 
The Raman spectra of the precursor- and wet-RGOCPDs are identical, with the D- and G-bands at 1347 and $1592 \mathrm{~cm}^{-1}$, respectively (Fig. 2a). Their large full width at half maximum (FWHM), in conjunction with a high D- to G-band intensity ratio, are indicative of a low degree of graphitization. This contrasts with the spectrum of graphite which has a small D-band and a much narrower G-band. Furthermore, the presence of a 2D-band at $2689 \mathrm{~cm}^{-1}$ in the graphite spectrum corroborates a high degree of graphitization. The XRD patterns of the RGO materials exhibited two broad peaks at $25^{\circ}$ and $44^{\circ}$, assigned to the (002) and (100) planes, respectively (Fig. 2b). The (002) peak position, shifted towards a lower angle with respect to that of graphite (at $27^{\circ}$ ), correlates with an increased interlayer distance while its large FWHM implies the predominance of small crystallites. Altogether, no measurable changes were observed by wetting the RGOCPD, confirming that the solvents used did not affect the structure and texture of the graphite crystallites in the active material.

a)

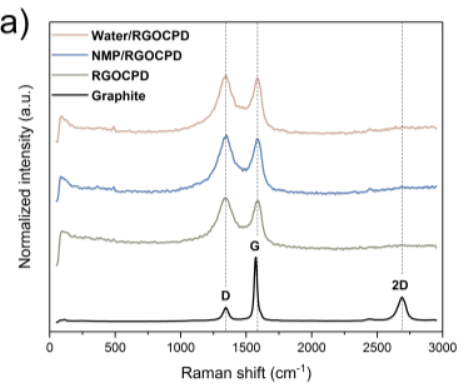

c)

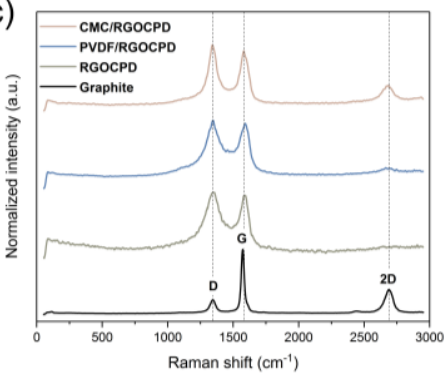

b)

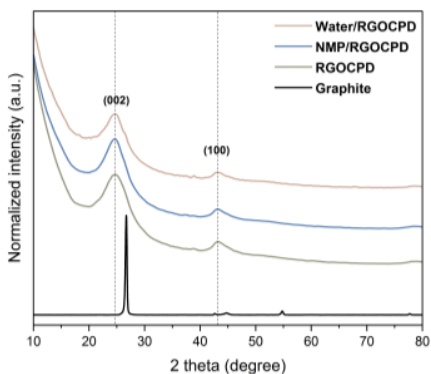

d)

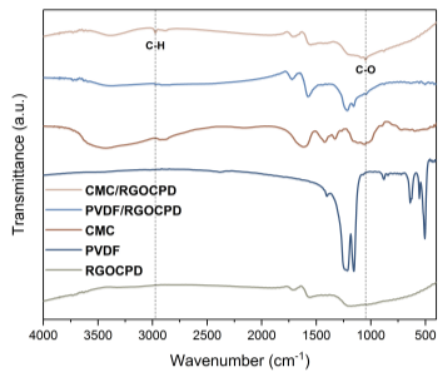

Figure 2. Materials characterization of RGOCPD materials: a) Raman spectra and b) XRD patterns; c) Raman spectra of the precursors, the PVDF/RGOCPD and the CMC/RGOCPD; d) FTIR of PVDF/RGOCPD and CMC/RGOCPD.

Next, we investigated the role of the binder/solvent pair. Again, strong D- and G-bands were identified in the Raman spectra of all RGO-containing samples (Fig. 2c). Remarkably, and while there were no major differences between the RGOCPD and the PVDF/RGOCPD, the G-band of the CMC/RGOCPD was redshifted by $\sim 10 \mathrm{~cm}^{-1}$. Moreover, the separation of the D- and G-bands is clearer in the CMC-based material (as the peaks are narrower). Together with a notable increase in the 2D-band intensity at $2684 \mathrm{~cm}^{-1}$, these observations mean that the $\mathrm{CMC} /$ water pair has acted on the texture of the RGOCPD. To clarify this interaction, we performed a FTIR analysis (Fig. 2d). The RGOCPD spectrum exhibited the typical fingerprint of these materials with broad peaks attributed to the stretching vibrations of $\mathrm{C}=\mathrm{O}\left(1706 \mathrm{~cm}^{-1}\right), \mathrm{C}=\mathrm{C}\left(1562 \mathrm{~cm}^{-1}\right)$ and $\mathrm{C}-\mathrm{OH}$ $\left(1179 \mathrm{~cm}^{-1}\right)$, respectively. [4] For the PVDF, several intense peaks were seen originating mostly from the C-F bending and out-of-plane deformation of different polymorphs (500 $-1300 \mathrm{~cm}^{-1}$ ), in addition to the faint signals at $1403 \mathrm{~cm}^{-1}$, assigned to the $\mathrm{CH}_{2}$ wagging vibration. [5] When mixed with the RGOCPD, most of these peaks got suppressed (only the $\mathrm{C}-\mathrm{F}$ vibration modes in the $1250-1150 \mathrm{~cm}^{-1}$ region were visible). This is expected 
considering the low concentration of binder in the PVDF/RGOCPD composite and, along with the absence of shifts or new peaks, points to a mostly physical mixture of the two components. As for the CMC, its spectrum showed a broad peak assigned to the $\mathrm{O}-\mathrm{H}$ stretch (centered at $3430 \mathrm{~cm}^{-1}$ ) with a shoulder derived from $\mathrm{C}-\mathrm{H}$ stretching (centered at $\left.2930 \mathrm{~cm}^{-1}\right)$. The $\mathrm{C}=\mathrm{O}\left(1620 \mathrm{~cm}^{-1}\right)$ and $\mathrm{C}-\mathrm{O}\left(1060 \mathrm{~cm}^{-1}\right)$ stretching vibrations, together with the $\mathrm{C}-\mathrm{H}$ rocking $\left(1421 \mathrm{~cm}^{-1}\right)$ and bending $\left(1328 \mathrm{~cm}^{-1}\right)$ modes complete the set of observed peaks. The CMC/RGOCPD composite shows the RGOCPD signals, with a curious sharpening of the $\mathrm{C}-\mathrm{H}$ and $\mathrm{C}-\mathrm{O}$ peaks (at $2950 \mathrm{~cm}^{-1}$ and $1060 \mathrm{~cm}^{-1}$, respectively). This is, possibly, a consequence of intermolecular interactions between the functional groups of RGOCPD and CMC and could explain the strong synergy between them (Fig. 2c). Moreover, it is likely that aqueous CMC acts as a strong dispersant of RGOCPD crystallites/flakes, compromising the porosity besides causing partial exfoliation and restacking. [6]

Lastly, we studied the interaction of the binders with the electrolyte. As seen in Fig. 3, when PVDF was added to $\mathrm{AlCl}_{3}:[\mathrm{EMIm}] \mathrm{Cl}=1.3\left(\mathrm{~mol} \mathrm{~mol}^{-1}\right)$, the ionic liquid darkened and the powdered binder was, mostly, left floating on top. Even after 1 hour, the electrolyte did not completely wet the PVDF implying a limited interaction between the two. By contrast, the CMC was rapidly dissolved. Logically, this raises the hypothesis that while PVDF retains its structural integrity when in contact with the electrolyte, the CMC dissolves completely in the electrolyte, resulting in a "leach-out" effect. This would compromise the charge transport as well as the stability of the electrode and/or electrolyte.

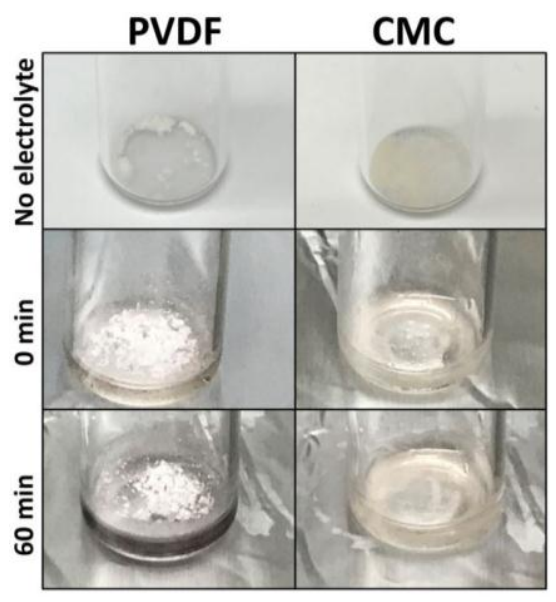

Figure 3. Visual assessment of the interaction of $\mathrm{PVDF}$ and $\mathrm{CMC}$ with $\mathrm{AlCl}_{3}:[\mathrm{EMIm}] \mathrm{Cl}=1.3\left(\mathrm{~mol} \mathrm{~mol}^{-1}\right)$.

Overall, PVDF does not seem to significantly affect the electrochemical performance of the RGO-based electrode. On the other hand, there is a complex interaction between the $\mathrm{CMC}$ and the rest of the $\mathrm{ACB}$ components. At present, it is not clear what compromises the electrochemical response of the CMC/RGOCPD electrode, but the following are some of the possible reasons:

- Dissolution of the CMC in chloride-based ionic liquids (IL) results in intermolecular interactions between the IL anions and the hydroxyl groups of the polymer, compromising the electrode-electrolyte charge transfers [7]

- The CMC coats the surface of carbonaceous materials in IL media [8], therefore blocking the mesopores of the RGOCPD and other redox active sites. 
- Cross-linking and gelling of CMC in the presence of chloroaluminate ions, compromising the electrode integrity [9]

- Electrolyte degradation by the introduction of residual moisture in the moisture-sensitive IL (due to CMC's strong water affinity)

While more work is needed to pinpoint the exact reasons for such different electrochemical behaviours, it is obvious that $\mathrm{CMC}$ is not a suitable binder for ACBs with chloride-based IL electrolytes.

\section{CONCLUSION}

The effect of PVDF and CMC binders on the electrochemical response of mesoporous reduced graphene oxide (RGOCPD) cathode for ACBs was studied. While PVDF enabled one of the highest capacities reported for carbonaceous materials, CMC degraded its performance considerably. This was attributed to the interaction between $\mathrm{CMC}$ and the chloride-based electrolyte.

\section{REFERENCES}

[1] J. Smajic, A. Alazmi, S. Patole and P. M. F. J. Costa, RSC Advances, 7(63), 39997-40004 (2017)

[2] J. Smajic, A. Alazmi, N. Batra, T. Palanisamy, D. H. Anjum and P. M. F. J. Costa, Small, $1803584(2018)$

[3] A. Alazmi, O. Tall, S. Rasul, M. N. Hedhili, S. P. Patole and P. M. F. J. Costa, Nanoscale 8(41), 17782-17787 (2016)

[4] A. Alazmi, S. Rasul, S. P. Patole and P. M. F. J. Costa, Polyhedron, 116, 153-161 (2016)

[5] Y. Bormashenko, R. Pogreb, O. Stanevsky and E. Bormashenko, Polymer Testing, 23(7), 791-796 (2014)

[6] K. Adarsh and S. Ramaprabhu, AIP Advances 2(3), 032183 (2012)

[7] S. Hina, Y. Zhang and H. Wang, Reviews on Advanced Materials Science, 40, 215-226 (2015)

[8] T. Zhao, L. Liu, G. Li, L. Du, X. Zhao, J. Yan, Y. Cheng, A. Dang and T. Li, Chinese Science Bulletin 57(14), 1620-1625 (2012)

[9] M. Anna, A. Laskowska, G. Boiteux, M. Zaborski, O. Gain and A. Serghei, Macromolecular Symposia, 341(1), 7-17 (2014) 